\title{
Study on Influencing Factors of Urban Residents' Winter Sports Consumption Behavior
}

\author{
Chuanming Jiao \\ Qiqihar Medical University, Qiqihar, Heilongjiang, 161006
}

Keywords: urban resident winter sports; consumption behavior; influencing factors

\begin{abstract}
Since the reform and opening-up, sports consumption has become more and more irreplaceable in China's economic construction. Nowadays, sports consumption has become an indispensable part of household consumption, which is in line with the trend of people's consumption structure. Through the discussion and research on the sports consumption of urban residents in China, this paper aims to understand the current situation and the factors of the consumption of residents in the cities and towns of our country, and provide scientific and reasonable suggestions for the construction of reasonable residents' sports consumption.
\end{abstract}

\section{Introduction}

Sports consumption is an economic activity in which people use sports to purchase sports utility. It is the premise and development motivation of the sports industry. After the reform and opening up, sports consumption, as an economic phenomenon, has risen and is gradually active in China. At present, sports consumption has become an important part of household consumption, and it is in line with the development trend of consumption structure upgrading. With the development of the national economy, sports consumption will gradually increase. This paper tries to make a rough discussion on the sports consumption of urban residents in China [1].

\section{Research Objects and Methods}

In order to analyze the factors influencing the winter sports consumption behavior of urban residents, our research team conducted a questionnaire survey on the residents walking in the Mudanjiang People's Park and Youth Park on November 5, 2006 (issuing the questionnaire and signing the receipt) Envelopes of addresses and stamps), 600 questionnaires were distributed, and 526 valid questionnaires were returned, with an effective recovery rate of $87.77 \%$. The research subjects considered factors such as gender, age, occupation and education level.

According to the content, purpose and tasks of this study, more than 40 articles (parts) on consumer literature were examined in detail, and they were effectively analyzed and utilized, and questionnaires were designed. The questionnaire was reviewed by three sports sociologists in Mudanjiang City. The structure validity and content validity of the questionnaire were tested. The experts confirmed the problem of the questionnaire, and the reliability of the questionnaire was tested [2].

\section{Factors Affecting Urban Residents' Winter Sports Consumption Behavior}

In the economic life of the whole society, as with other consumer behaviors affecting the public, the factors that restrict and affect the winter sports consumption of urban residents in Mudanjiang are mainly the economic conditions of the society and the residents themselves. The survey results show that the factors that contribute to the promotion of urban residents' winter sports consumption behavior in the top three are: positive sports consumption concept, stable income and more leisure time; and factors restricting urban residents' winter sports consumption. The order of the top three is that the economic income level is low, the lack of perfect physical fitness facilities and sports consumption prices are higher. 
The survey results of sports service attitudes of different income groups in cities show that there is a close relationship between income level and sports fitness and entertainment consumption awareness and attitude. The higher the proportion of consumption in the place, the more than half of it; and the higher the per capita income level of the family, the higher the proportion of people who are willing to pay for the fitness and entertainment places, especially when the monthly income of the family reaches more than 3,000 yuan. The proportion of residents who are willing to pay for paid fitness has reached $22.03 \%$, which is far higher than the preference ratio of other income levels. It can be seen that if consumer credit is not considered, the income level is the main factor affecting residents' winter sports consumption [3].

In addition to the income level, the price factor is also a hard constraint that restricts the consumption of residents' sports services. 36. 6\% of consumers think that the price of local fitness and entertainment venues is high; $34.9 \%$ of consumers think that the price of local sports events is high. 42. 8\% of consumers believe that the consumer price of sports and fitness venues can be less than 10 yuan each time; 25 . $2 \%$ of consumers believe that the price of live sports watching can be sustained at 10 . Too expensive tickets or high fitness venues are the third most important factor limiting consumer spending. If consumer spending is divided into consumer necessities and luxury spending, sports service consumption is a luxury expenditure, as luxury goods have greater demand price elasticity, coupled with instability in future income expectations, will intensify sports services. The marginal consumption of luxury goods is reduced, which further leads to insufficient effective market demand and restricts the level of sports consumption.

Effective sports demand includes not only the disposable income, the desire for sports demand, but also a certain amount of leisure time. Drawing on the leisure time model proposed by labor economists, for consumers to get more consumption, he must give up his leisure time, which means he can earn more money and buy more goods and services. Especially for residents in economically underdeveloped areas, people have to use all the time to produce labor in order to survive. It is not difficult to understand that sports service consumption is essentially a consumption time activity, and the lack of leisure time is one of the key factors restricting residents' consumption of sports services, and there are significant differences between different ages. The lack of leisure time makes more residents aged 30-39 and 40-49 years away from sports service consumption. This is in line with many literatures showing that China's sports population is mainly concentrated in the adolescent stage and the old age stage.

Sports consumers of different consumption methods have strongly reflected the choice of the geographical location of the stadium. This is related to the inconvenience caused by the lack of urban sports facilities, and the relative concentration and unreasonable distribution pattern. At present, the lack of urban sports facilities has become one of the main constraints affecting residents' winter sports activities. Due to the long distance and inconvenient transportation, most sports consumers are troubled by choosing a time-saving and convenient suitable place. Therefore, at this stage, the residents of our city attach importance to the choice of venues and their objective conditions.

Sports consumption is also influenced by personal characteristics, especially by the personality, occupation, and education level of consumers. The analysis of consumer motivations of respondents with sports service consumption behaviors shows that personal interests and hobbies are one of the important factors influencing their consumption of sports services. Especially for physical fitness activities, it is still necessary to obtain the joy of success through continuous practice of movement skills. Among them, there is no lack of willpower and hard work, and if the interest in sports consumption is only one, his hobbies are extremely easy. Transfer quickly. Through the comparative analysis of the attitudes of sports service consumption of different professional consumers, it can be seen that there are significant differences between the sports consumption attitudes of different professional consumers. For this sorting, an interactive analysis of occupational and family annual per capita income shows that the differences exhibited by different occupational groups are still closely related to their income levels. The degree of consumer culture education has the most extensive and far-reaching impact on consumer behavior. Through a comparative analysis of 
consumer attitudes at different levels of culture, there is a clear positive correlation between the level of education and the attitude of sports service consumption attitudes. That is, as the level of education increases, the proportion of consumers tending to consume sports services Increased, the specific order is graduate students or graduate students, high school or secondary school, junior high school, and below primary school [4].

\section{Measures to Expand the Sports Consumption of Urban Residents in China}

Residents' income is the basis of household consumption. The increase in income can promote the rapid growth of consumption. The decline in income will inevitably lead to a decline in consumption. According to relevant surveys, since 1995, the income growth rate of Chinese residents has been lower than the growth rate of GDP. This affects household consumption in two ways. First, the slowdown in income growth has directly hampered consumption growth. Second, the slowdown in income growth has led to a decline in household income expectations, which indirectly inhibits consumption growth. Therefore, to increase sports consumption, we must first solve the problem of increasing residents' income. Of course, increasing the income of residents is a category of macroeconomic adjustment and requires the government to solve the problem. If the problem of household income can be solved well, the popularization of sports consumption in China will develop rapidly.

The school is an important place to train a variety of professionals. In the process of expanding sports consumption, we must pay attention to the role of school sports. It is necessary to cultivate people's concept of lifelong sports, thereby increasing the number of sports population of urban residents in China. School physical education must adapt to the needs of the society and cultivate specialized and multi-functional sports backbone needed by the society so that they can become the dominant players in school sports and become social sports instructors, thus further satisfying the needs of residents' sports activities and improving sports activities. We should learn from the experience of developed countries and rely on the mass sports activities to be carried out in school sports venues. This will not only meet the needs of mass sports, but also promote the effective use of school sports facilities and make up for the shortage of social sports facilities [5].

At present, the insufficient number of sports facilities is an important factor affecting the sports consumption of urban residents in China. Therefore, it is necessary to strengthen the construction of sports facilities as soon as possible. In addition to the country's continued increase in investment, market mechanisms should also be used to guide non-state-owned investors to build multi-functional, small-scale and suitable physical fitness and entertainment venues in communities and residential communities to create urban residents. The current focus of developing China's sports market should be placed in the sports goods, market, fitness and entertainment market and competition performance market. The sporting goods market is a market for sports material products. In recent years, as people's health awareness has increased, more and more people have participated in various fitness activities. Sporting goods, especially sportswear, shoes and hats, fitness equipment and some sports and leisure products, have maintained a hot trend in the market. A number of well-known brands such as "Li Ning”, "Conway”, "Gweit”, “ "The British Paez", "Double Star", etc., stand out. In the future, the sports goods market should develop rapidly. It is necessary to speed up the development of new products, continuously improve the quality of sporting goods, and realize the serialization of products and styles to meet the diverse needs of consumers. The fitness and entertainment market and the competition show market are the core markets for sports consumption. To expand sports consumption, these two markets must be perfected. First of all, the relevant departments should put the entry point of fitness and entertainment consumption into the community sports service, and take the community fitness club as the organizational form, the group chain operation as the marketing method, and combine the sports service facilities and service environment to adjust the cost. To provide quality services at a price acceptable to the average consumer, so that fitness and entertainment consumption will be developed to the masses. Secondly, the competition and performance of competitive sports, as an activity with viewing characteristics, whether it is in person or on the screen, has become an 
indispensable part of the leisure life of urban residents.

\section{Conclusion}

Any industry needs to be marketized. Its development premise and economic premise are that the population of consumer products must be popularized. Therefore, we can consider implementing a low-cost strategy acceptable to most consumers, and develop popular sports such as skating, skiing, basketball, table tennis, martial arts, and fitness dance.

\section{Acknowledgements}

Fund Project: "Study on the Consumption Concept and Behavior Cultivation of Winter Ice and Snow Sports for Urban Residents in Qiqihar City", the subject belongs to: Qiqihar Philosophy and Social Science Basic Theory Research Project

No.: QSX2016-47JL

\section{References}

[1] Mao Sulin, Huo Zhao. A brief discussion on the cost of living in the city of China [J]. Shanxi University of Finance and Economics, 2001 (6).112

[2] Cui Haiyan. Current Situation and Prospects of Sports Consumption of Urban Residents in China [J]. Xi'an Institute of Physical Education, 2007.12

[3] Zhai Rui. Research on the status quo and the factors of sports consumption of urban residents in China [J]. Science and Technology Information, 2008.56

[4] Kong Wenqing, Chen Zhuoyuan. Investigation and Research on the Consumption Level of Residents in Urban Areas of China [J]. Journal of Beijing Sport University, 2002(6).67

[5] Chen Ruibi. Analysis of the Restrictive Factors of Sports Consumption in China [J]. Journal of Hubei Radio and Television University, 2007(6).58 